\title{
Clinical implication of HLA class I expression in breast cancer
}

\author{
Koichi Kaneko', Sumiya Ishigami ${ }^{1 *}$, Yuko Kijima', Yawara Funasako ${ }^{1}$, Munetsugu Hirata' ${ }^{1}$, Hiroshi Okumura', \\ Hiroyuki Shinchi ${ }^{1}$, Chihaya Koriyama ${ }^{2}$, Shinichi Ueno ${ }^{1}$, Heiji Yoshinaka ${ }^{1}$ and Shoji Natsugoe ${ }^{1}$
}

\begin{abstract}
Background: Human leukocyte antigen (HLA)-class I molecules on tumor cells have been regarded as crucial sites where cytotoxic T lymphocytes (CTL) can recognize tumor-specific antigens and are strongly associated with antitumor activity. However, the clinical impact of HLA class I expression in breast cancer has not been clarified.

Methods: A total of 212 breast cancer patients who received curative surgery from 1993 to 2003 were enrolled in the current study. HLA class I expression was examined immunohistochemically using an anti-HLA class I monoclonal antibody. The correlation between HLA class I positivity and clinical factors was analyzed.

Results: The downregulation of HLA class I expression in breast cancer was observed in 69 patients (32.5\%). HLA class I downregulation was significantly associated with nodal involvement $(p<0.05)$, TNM stage $(p<0.05)$, lymphatic invasion $(p<0.01)$, and venous invasion $(p<0.05)$. Patients with preserved HLA class I had significantly better disease-free interval (DFI) than those with loss of HLA class I ( $p<0.05)$. However, in multivariable analysis, HLA class I was not selected as one of the independent prognostic factors of disease-free interval.
\end{abstract}

Conclusion: The examination of HLA class I expression is useful for the prediction of tumor progression and recurrent risk of breast cancer via the antitumor immune system.

Keywords: HLA class I, survival, T cell immunology, antitumor activity

\section{Background}

The antitumor activity via cytotoxic T lymphocytes (CTL) or tumor antigen has been clarified in the oncological field. Activation of anti-tumor CTL requires the recognition of immunogenic epitopes presented on various types of human leukocyte antigen (HLA) class I molecules on the tumor [1-4]. The concept of immune surveillance is maintaining the relationship between tumor-associated antigens (TAA) complexing with the HLA class I and tumor-specific cytotoxic $\mathrm{T}$ cells. These activated tumor-specific cytotoxic $\mathrm{T}$ cells can eliminate cancer cells specifically. The loss of HLA class I on the tumor is believed to lead to malfunction of recognition by the CD8+ T cells. It is already known that malignancies exhibit altered or lost expression of histocompatible antigens on the tumor cells [5-7]. Loss of HLA class I antigens appears to be a significant mechanism by which

\footnotetext{
* Correspondence: ishiga@m.kufm.kagoshima-u.ac.jp

'Department of Surgical Oncology, Breast and Endocrine Surgery, Kagoshima University School of Medicine, Kagoshima, Japan Full list of author information is available at the end of the article
}

tumor cells escape specific immune attack and causes problems in the design of antitumor immunotherapy [7]. The loss of HLA class I antigens on tumor cells has been reported in several human tumors [5-7], and the loss of HLA class I molecules has been discussed in the context of tumor aggressiveness, such as differentiation of histology [8-10], invasiveness, and metastatic potential [5,11].

The non-covalent association with $\beta$ 2-microglobulin ( $\beta$ $2 \mathrm{~m}$ ) is essential for the structural stability and optimal function of HLA class I molecules [12]. Thus, several authors have used immunostaining of $\beta 2 \mathrm{~m}$ for the analysis of overall surface expression of HLA class I molecules $[9,11,13]$. However, there are often difficulties in evaluating immunostaining using anti-MHC class I monoclonal antibodies (mAbs), such as W6/32, $\mathrm{HC}-10$, or $\mathrm{HC}-\mathrm{A} 2$, as these type of antibodies are not ideal for the immunostaining of formalin-fixed, paraffin-embedded tissue. Moreover, these antibodies are not fully recognized whole HLA class I properly. Recently, EMR8-5, a monoclonal antibody against HLA class I heavy chains (HLA-A, B, C), has been
C Biomed Central 
validated in HLA class I immunohistochemistry [14-16], and used to investigate HLA class I expression in osteosarcoma [14], non-small cell lung cancer [15], and renal cell carcinoma [16]. The clinical implication of the HLA class I expression has been discussed and reviewed in breast cancer and it is not clarified [17-21]. Moreover, there have been no studies on HLA class I expression of breast cancer by EMR8-5. The present study assessed HLA class I expression in invasive breast cancer by immunohistochemistry using the EMR8-5 antibody, analyzed associations with clinicopathological factors, and discussed the clinical implication of HLA class I-positive breast cancer.

\section{Methods}

\section{Patients}

A total of 212 breast cancer patients, who consecutively underwent curative operation for primary invasive breast cancer at Kagoshima University Hospital from 1993 and 2003, were enrolled in the current study. None of the patients received any preoperative systemic chemotherapy or endocrine therapy. Clinicopathological features were documented according to TNM classification [17]. All patients were female and their mean age was 56 (ranging from age 23 to 90). One hundred and fifty-three underwent mastectomy and the remaining 59 underwent partial resection. A total of 84 patients had nodal involvement, and the numbers of patients with stages I, II, and III were 70,101 , and 41 , respectively. This study was approved by the Ethical Committee of the University of Kagoshima, and written informed consent was obtained from all individuals. Overexpression of the estrogen receptor (ER), progesterone receptor (PgR), and HER2 were examined by immunohistochemical staining using the appropriate primary antibodies. Distinct staining of the nucleus in more than $10 \%$ of tumor cells was recorded as positive for ER and PgR, and strong membrane staining in more than $10 \%$ of invasive lesions was recorded as positive for HER2.

\section{Immunohistochemical analysis of HLA class I in breast cancer}

HLA class I expression was investigated by immunohistochemical staining with the monoclonal anti-pan HLAclass I antibody 5 EMR8-5 (Cosmo Bio Co., Tokyo, Japan). EMR8-5 is an anti-pan HLA class I monoclonal antibody, which can recognize all of HLA A, B, and C heavy chain even in formalin-fixed tissue [22].

The avidin-biotin complex $(\mathrm{ABC})$ method was used to visualize HLA class I expression in breast cancer. Human tonsil sections were used as positive controls for HLA class I, and in the negative controls, the primary antibody was replaced with buffer. The ABC method was performed in accordance with previous reports [14-16]. Namely, $4 \mu \mathrm{m}$ paraffin-embedded sections of breast cancer were de-paraffinized and soaked in PBS. The sections were treated with $3 \% \mathrm{H}_{2} \mathrm{O}_{2}$ for $30 \mathrm{~min}$ in order to block endogenous tissue peroxidases, followed by treatment with rabbit serum for $60 \mathrm{~min}$ in order to reduce non-specific binding. Primary anti-HLA-class I antibody was diluted to 1:100 and incubated with the tonsil sections at $4^{\circ} \mathrm{C}$ overnight. Sections were rinsed in PBS and visualized using standard techniques for labeled avidin-biotin immunoperoxidase staining.

All specimens were reviewed independently using light microscopy for at least five areas at a $400 \times$ magnification by two investigators (KK and SI) who were blinded with respect to the clinicopathological data. The intensity of HLA-class I staining was evaluated in accordance with a previous report [23], using the following criteria: strongly positive (positive), defined as complete membrane staining in $40 \%$ or more of tumor cells; weakly positive (negative), any lesser degree of staining appreciable in tumor cells; and absent (negative), no appreciable staining in tumor cells.

\section{Survival analysis and statistical evaluation}

Postoperative intervals were estimated by the KaplanMeier method. In the survival analysis, we excluded the cases that had not undergone curative resection. The endpoint of survival analysis was defined as the day of death of each patient from not only cancer-related events but also other causes. That of disease-free survival (DFS) was defined as a locoregional recurrence or distant metastasis of breast cancer in soft tissue, lymph nodes, liver, lung, brain, and/or bone by physical and/or pathological examination. Overall survival (OS) and DFS of 212 patients were $88.4 \%$ and $83.0 \%$, respectively. Median and mean survivals of these patients were 71.9 and 76.2 months, respectively.

Significant differences of DFS and OS were calculated using the log-rank test, and significant differences in categorical variables were analyzed by the $\chi^{2}$-test. Univariate and multivariable analyses of the postoperative outcome were conducted using Cox's proportional hazards model. Differences were considered significant at $\mathrm{p}<0.05$. All statistical analyses were performed using Stat View 5.0 software.

\section{Results}

Expression and evaluation of HLA class I in breast cancer tissue

HLA class I positivity was found in not only the membrane of tumor cells but also in the cytoplasm of tumor cells (Figure 1). In addition, Some stromal lymphocytes also showed HLA class I positivity. Generally, there was little HLA class I positivity in normal mammary glands adjacent to cancerous tissue. According to the previously mentioned evaluation, 69 patients $(32.5 \%)$ had strong HLA class I expression, 64 patients (30.2\%) had weak 


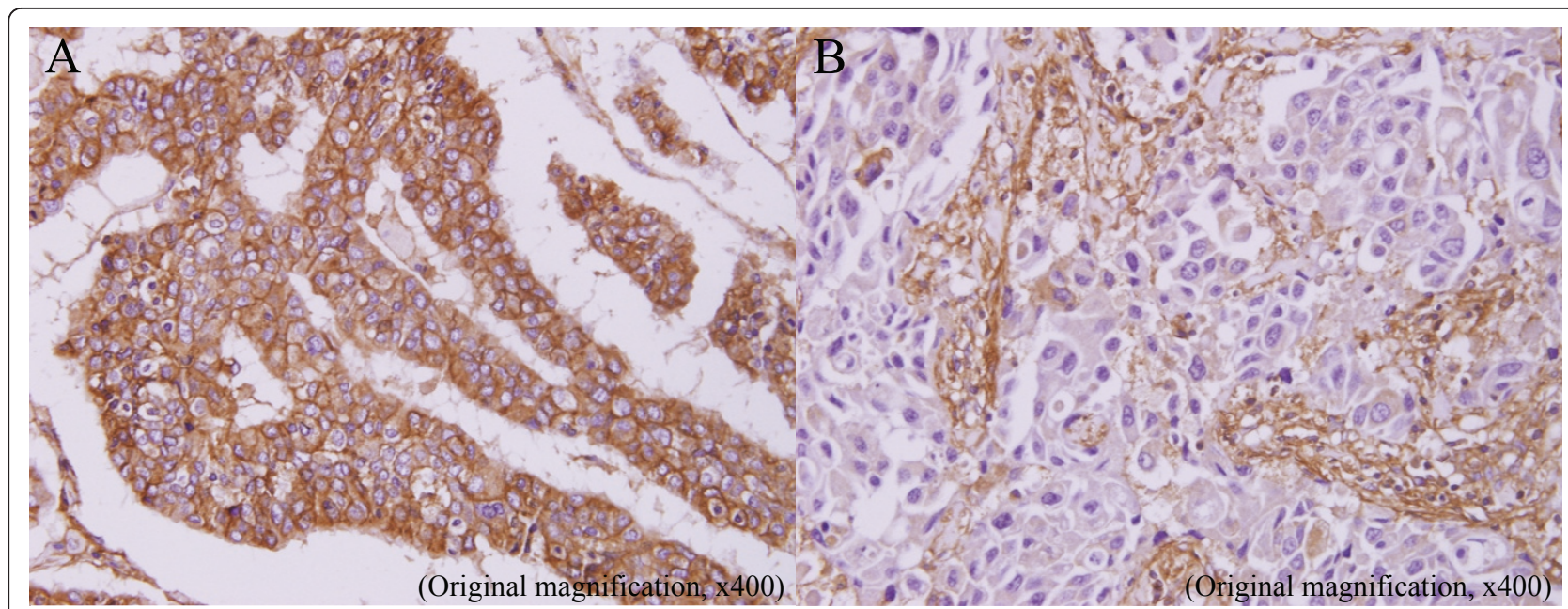

Figure 1 Representative images of immunostaining of HLA class I in invasive breast cancer. A: positive expression, B: downregulated expression.

expression, and the remaining 79 patients (37.3\%) lacked expression. Patients with weak or no expression of HLA class I antigens in breast cancer were classified as the downregulated HLA class I group. In contrast, patients with more than $40 \%$ of HLA class I positivity were classified as the positive HLA class I group.

\section{Association between HLA class I and clinicopathological factors}

The downregulation of HLA class I expression in breast cancer was significantly associated with nodal metastasis, TNM, lymphatic invasion, and venous invasion $(\mathrm{p}=0.04$, $\mathrm{p}=0.01, \mathrm{p}=0.006$, and $\mathrm{p}=0.04$, respectively) (Table 1 ). There was no significant association between HLA class I expression and histology or hormonal status.

Patients' overall survival (OS) and disease-free survival (DFS) with or without HLA class I expression in breast cancer

Postoperative OS was not significantly different according to HLA class I expression. However, patients with HLA class I positivity had significantly longer DFS than those without HLA class I positivity $(\mathrm{p}<0.05)$ (Figure 2$)$. Using univariate analysis, seven clinical factors, including HLA class I expression, were selected as significant for DFS (Table 2). According to multivariable analysis using these seven factors, lymph node metastasis, progesterone receptor, and vascular invasion were independent prognostic factors. HLA class I expression was not selected as an independent factor for DFS (Table 2).

\section{Discussion}

The monoclonal antibody EMR8-5 can recognize all of HLA A, B, and C heavy chain even in formalin-fixed tissue. In this context, EMR8-5 can recognize whole HLA molecules, and its validity was supported by the immunostaining performed in our current studies. Cordon et al. reported that HLA class I positivity examined using the conventional HLA class I antibody W6/32, which also recognizes all HLA class I antigens, was 30\% of HLA class I positivity in breast cancer, similar to that shown in our present study [24]. In contrast, Madjd et al. investigated HLA class I expression in breast cancer using a HC-10 antibody [24], and demonstrated that HLA class I negativity correlated with a better postoperative outcome. These results conflicted with the data in our studies. This discrepancy may be explained by the fact that whereas the $\mathrm{HC} 10 \mathrm{mAb}$ scarcely reacts with HLA-A alleles, the anti- HLA class I heavy chain mAb EMR8-5 can detect all recombinant proteins of HLA-A, B, and C alleles by immunoblot analysis [25]. In addition, EMR8-5 can be applied to paraffin-fixed specimens, so in this context it is an ideal antibody for evaluating cancerous HLA class I antigen expression.

In our current study, the downregulation of HLA class I expression in breast cancer was $66 \%$, which was more than that in gastric (32\%) [22] and esophageal cancer (43\%) [26], and osteosarcoma (55\%) [14], but less than the downregulation in lung cancer (70\%) [15]. The degree of HLA class I loss may be affected by organ specificity. For example, Ishigami et al. speculated that highly preserved HLA class I expression in gastric cancer is partly due to exogenous stimulation from gastritis or bacterial infection of Helicobacter pylori [22]. The differences in HLA class I expression in breast cancer may also be explained by the possible inflammation and proteolysis that can occur at the sites of breast cancer origin. These are important steps linked both to HLA loss and cancer aggressiveness. In this study, there was little HLA class I positivity in normal 
Table 1 Association between clinical factors and HLA class I expression in 212 breast cancer patients

\begin{tabular}{|c|c|c|c|}
\hline & Positive $(n=69)$ & Negative $(n=143)$ & $p$ value \\
\hline \multicolumn{4}{|l|}{ Age (years) } \\
\hline$<50$ & $23(11 \%)$ & $59(28 \%)$ & 0.2669 \\
\hline $51<$ & $46(22 \%)$ & $84(40 \%)$ & \\
\hline \multicolumn{4}{|l|}{ Tumor size } \\
\hline $\mathrm{T} 1$ & $35(17 \%)$ & $51(24 \%)$ & 0.0641 \\
\hline $\mathrm{T} 2$ & $31(15 \%)$ & 72 (34\%) & \\
\hline $\mathrm{T} 3$ & 1 & $12(57 \%)$ & \\
\hline $\mathrm{T} 4$ & 2 & $8(4 \%)$ & \\
\hline \multicolumn{4}{|l|}{ Nodal invasion } \\
\hline Negative & $49(23 \%)$ & 79 (37\%) & 0.0278 \\
\hline Positive & $20(9 \%)$ & $64(30 \%)$ & \\
\hline \multicolumn{4}{|l|}{ Estrogen receptor } \\
\hline Negative & $18(8 \%)$ & $53(25 \%)$ & 0.1126 \\
\hline Positive & $51(24 \%)$ & $90(42 \%)$ & \\
\hline \multicolumn{4}{|l|}{ Progesterone receptor } \\
\hline Negative & $25(12 \%)$ & $64(30 \%)$ & 0.2387 \\
\hline Positive & $44(21 \%)$ & 79 (37\%) & \\
\hline \multicolumn{4}{|l|}{ Lymphatic invasion } \\
\hline Negative & $46(22 \%)$ & 67 (32\%) & 0.0067 \\
\hline Positive & $23(11 \%)$ & 76 (36\%) & \\
\hline \multicolumn{4}{|l|}{ Venous invation } \\
\hline Negative & $66(31 \%)$ & $122(58 \%)$ & 0.0260 \\
\hline Positive & $3(1 \%)$ & $21(10 \%)$ & \\
\hline \multicolumn{4}{|l|}{ TNM } \\
\hline I & $31(15 \%)$ & $39(18 \%)$ & 0.0110 \\
\hline$\|$ & $31(15 \%)$ & 70 (33\%) & \\
\hline III & $7(3 \%)$ & $34(16 \%)$ & \\
\hline \multicolumn{4}{|l|}{ Histology } \\
\hline IDC & $67(32 \%)$ & $142(67 \%)$ & 0.2040 \\
\hline ILC & 2 & 1 & \\
\hline \multicolumn{4}{|l|}{ HER2 receptor } \\
\hline Negative & $63(30 \%)$ & $123(58 \%)$ & 0.4539 \\
\hline Positive & $6(3 \%)$ & $17(8 \%)$ & \\
\hline
\end{tabular}

mammary gland tissue. In contrast, HLA class I antigens were preserved in early breast cancer, and cancerous HLA class I antigens were newly expressed or reduced according to the tumor extension. Although T1 tumors had $41 \%$ HLA class I positivity, T3-4 tumors only showed $15 \%$ positivity. According to tumor extension, preservation of HLA class I of the tumor was reduced. This clinical trait was also reported in other types of malignancies, such as gastrointestinal cancer [22,26] and sarcoma [14]. It is possible that, in the process of tumor extension, tumors that lost HLA class I survived and escaped from antigen-specific CTL-mediated lysis leading to tumor dissemination and metastasis. However, these results do not fully explain the relationship with metastasis, therefore we need to perform more analyses comparing the results among in situ, lobular and ductal breast cancers on key parameters, such as VEGF, MMP etc.
In the current study, the downregulation of HLA class I expression was significantly associated with lymphatic and nodal invasion. Mizukami et al. showed that when HLA class I-positive esophageal cancer metastasized to the lymph node, tumor cells completely lost HLA class I expression in this system [22]. Zia et al. investigated the immunological characteristics of isolated cancer cells (ITC) in bone marrow and found that ITCs with the HLA class I downregulation phenotype were often derived from poorly differentiated primary breast carcinomas which was associated with a short survival period in breast cancer [27]. Therefore, cancerous HLA class I downregulation seems to be conducive to metastasis to other organs.

Patients with positive HLA class I expression showed a better DFS in comparison with those with downregulation of HLA class I expression. This result differs from that for 


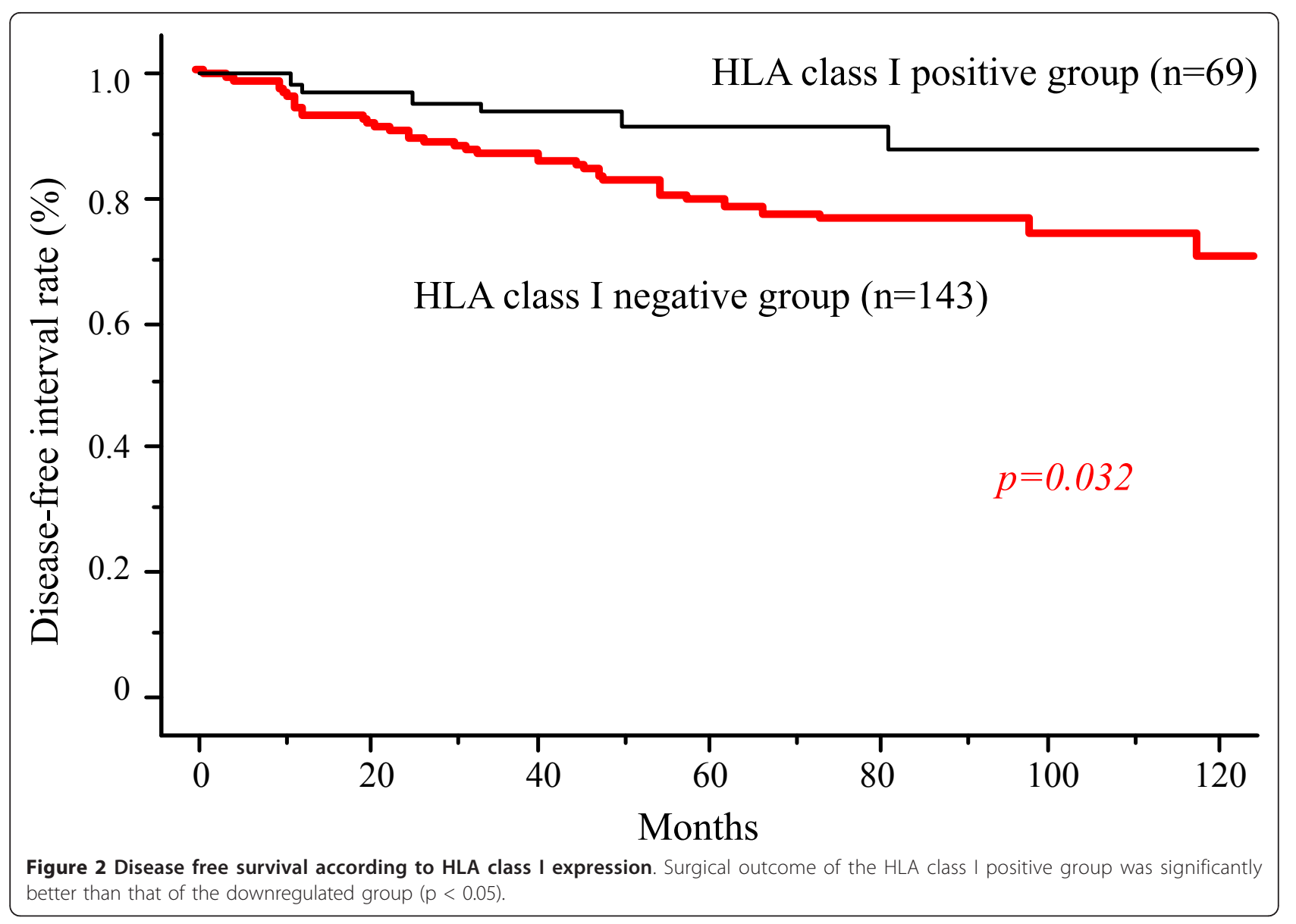

esophageal cancer [26]. In breast cancer, the average OS is generally better than those in other malignancies; DFS is often used to evaluate aggressiveness of biological markers in breast cancer. In this context, significant differentiation in DFS seems to be meaningful.

It has been clarified that HLA molecule inactivity depends not only on the expression of HLA class I molecules themselves, but also on the post-transcriptional course that mainly affects $\beta 2$-microglobulin gene expression. Aptsiauri et al. [28] showed that if apparent tumor cells expressed HLA class I, various types of HLA class I alterations were found in malignancies and in the molecular mechanisms that underlie these defects. In this context, the HLA class I molecules preserved in these breast cancers may exhibit altered expression and dysfunction as antigen presentation molecules. It seems to be difficult to precisely evaluate HLA class I expression, however, in the current study, we evaluate HLA expression including in $\beta 2$ microglobulin expression using the EMR8-5 antibody.

Table 2 Univariate and multivariable analyses for DFS

\begin{tabular}{|c|c|c|c|c|c|}
\hline \multirow[b]{3}{*}{ Variables } & \multirow[b]{3}{*}{ Categories } & \multicolumn{2}{|l|}{ Univariate analysis } & \multicolumn{2}{|l|}{ Multivariable analysis } \\
\hline & & \multicolumn{2}{|l|}{ Disease free interval } & \multicolumn{2}{|l|}{ Disease free interval } \\
\hline & & HR (95\% confidence interval) & $p$ value & HR ( $95 \%$ confidence interval) & $p$ value \\
\hline Tumor size & $\mathrm{T} 3+\mathrm{T} 4$ (vs T1+T2) & $0.249(0.120-0.514)$ & $P=0.0020$ & $0.459(0.212-0.995)$ & $P=0.0485$ \\
\hline Nodal involvement & N1 (vs N0) & $0.148(0.068-0.324)$ & $P<0.0001$ & $0.230(0.092-0.574)$ & $P=0.0016$ \\
\hline Estrogen receptor & Positive (vs negative) & $1.656(0.872-3.146)$ & 0.1231 & & \\
\hline Progesterone receptor & Positive (vs negative) & $1.932(1.019-3.664)$ & $P=0.0437$ & $2.168(1.109-4.240)$ & $P=0.0237$ \\
\hline Lymphatic invasion & Positive (vs negative) & $0.246(0.117-0.521)$ & $P=0.0020$ & $0.871(0.346-2.194)$ & 0.7689 \\
\hline Vascular invasion & Positive (vs negative) & $0.184(0.094-0.361)$ & $P<0.0001$ & $0.423(0.198-0.904)$ & $P=0.0264$ \\
\hline HER2 receptor & Positive (vs negative) & $0.653(0.272-1.565)$ & 0.3390 & & \\
\hline HLA class I expression & Negative (vs positive) & $2.513(1.050-6.012)$ & $P=0.0384$ & $1.691(0.696-4.110)$ & 0.2462 \\
\hline
\end{tabular}




\section{Conclusions}

The downregulation of HLA class I expression frequently occurred in breast cancer, in a similar manner to what has been seen in several other cancers, and it may also be associated with tumor progression and relapse. Therefore HLA class I status may be useful with other well-known prognostic factors like nodal involvement and hormone status to evaluate postoperative outcomes in breast cancer.

\section{Abbreviations}

ABC method: Avidin-biotin complex method; CTL: cytotoxic T lymphocytes; DFS: disease free survival; HLA: Human leukocyte antigen; OS: overall survival.

\section{Author details}

'Department of Surgical Oncology, Breast and Endocrine Surgery, Kagoshima University School of Medicine, Kagoshima, Japan. ${ }^{2}$ Department of Radiology, Graduate School of Medical and Dental Sciences, Kagoshima University, Kagoshima, Japan.

\section{Authors' contributions}

KY carried out the immuno histopathological studies and performed the statistical analysis. SI participated in its design and coordination. All authors read and approved the final manuscript.

\section{Competing interests}

The authors declare that they have no competing interests.

Received: 10 January 2011 Accepted: 20 October 2011

Published: 20 October 2011

\section{References}

1. Marincola FM, Jaffee EM, Hicklin DJ, Ferrone S: Escape of human solid tumors from T-cell recognition: molecular mechanisms and functional significance. Adv Immunol 2000, 74:181-273.

2. Tangri S, Ishioka GY, Huang X, Sidney J, Southwood S, Fikes J, Sette A: Structural features of peptide analogs of human histocompatibility leukocyte antigen class I epitopes that are more potent and immunogenic than wild-type peptide. J Exp Med 2001, 194:833-846.

3. Cabrera T, Maleno I, Collado A, Lopez Nevot MA, Tait BD, Garrido F: Analysis of HLA class I alterations in tumors: choosing a strategy based on known patterns of underlying molecular mechanisms. Tissue Antigen 2007, 69(Suppl 1):264-268.

4. Nagorsen D, Thiel E: HLA typing demands for peptide-based anti-cancer vaccine. Cancer Immunol Immunother 2008, 57:1903-1910.

5. Garrido F, Cabrera T, Concha A, Glew S, Ruiz-Cabello F, Stern PL: Natural history of HLA expression during tumour development. Immunol Today 1993, 14:491-499.

6. Garrido F, Ruiz-Cabello F, Cabrera T, Perez-Villar JJ, Lopez-Botet M, DugganKeen M, Stern PL: Implications for immunosurveillance of altered HLA class I phenotypes in human tumours. Immunol Today 1997, 18:89-95.

7. Hicklin DJ, Marincola FM, Ferrone S: HLA class I antigen downregulation in human cancers: T-cell immunotherapy revives an old story. Mol Med Today 1999, 5:178-186

8. Eyal A, Levin I, Segal S, Levi I, Klein B, Kuperman O: Variation of HLA-ABC surface antigen expression on adenocarcinoma of the colon in correlation with the degree of differentiation. Nat Immun Cell Growth Regul 1990, 9:222-227.

9. Levin I, Klein T, Goldstein J, Kuperman O, Kanetti J, Klein B: Expression of class I histocompatibility antigens in transitional cell carcinoma of the urinary bladder in relation to survival. Cancer 1991, 68:2591-2594.

10. Concha A, Cabrera T, Ruiz-Cabello F, Garrido F: Can the HLA phenotype be used as a prognostic factor in breast carcinomas? Int I Cancer Suppl 1991, 6:146-154

11. Petersen BL, Petersen $C L$, Braendstrup O, Mouritsen S, Engel AM, Svane IM, Werdelin O: Expression of beta 2-microglobulin by premalignant epithelium. APMIS 1993, 101:529-536.
12. Pedersen LO, Stryhn A, Holter TL, Etzerodt M, Gerwien J, Nissen MH, Thogersen $\mathrm{HC}$, Buus $\mathrm{S}$ : The interaction of beta 2-microglobulin (beta $2 \mathrm{~m}$ ) with mouse class I major histocompatibility antigens and its ability to support peptide binding. A comparison of human and mouse beta $2 \mathrm{~m}$. Eur J Immunol 1995, 25:1609-1616.

13. Cabrera T, Angustias Fernandez M, Sierra A, Garrido A, Herruzo A, Escobedo A, Fabra A, Garrido F: High frequency of altered HLA class I phenotypes in invasive breast carcinomas. Hum Immunol 1996, 50:127-134.

14. Tsukahara T, Kawaguchi S, Torigoe T, Asanuma H, Nakazawa E, Shimozawa K, Nabeta Y, Kimura S, Kaya M, Nagoya S, Wada T, Yamashita T, Sato N: Prognostic significance of HLA class I expression in osteosarcoma defined by anti-pan HLA class I monoclonal antibody, EMR8-5. Cancer Sci 2006, 97:1374-1380.

15. Kikuchi E, Yamazaki K, Torigoe T, Cho Y, Miyamoto M, Oizumi S, Hommura F, Dosaka-Akita H, Nishimura M: HLA class I antigen expression is associated with a favorable prognosis in early stage non-small cell lung cancer. Cancer Sci 2007, 98:1424-1430.

16. Kitamura H, Honma I, Torigoe T, Asanuma H, Sato N, Tsukamoto T: Downregulation of HLA class I antigen is an independent prognostic factor for clear cell renal cell carcinoma. J Urol 2007, 177:1269-1272.

17. Campoli M, Ferrone S: HLA antigen changes in malignant cells: epigenetic mechanisms and biologic significance. Oncogene 2008 27:5869-85.

18. de Kruijf EM, Sajet A, van Nes JG, Natanov R, Putter H, Smit VT, Liefers GJ, van den Elsen PJ, van de Velde CJ, Kuppen PJ: HLA-E and HLA-G expression in classical HLA class I-negative tumors is of prognostic value for clinical outcome of early breast cancer patients. J Immunol 2010, 185:7452-9.

19. Boomsma MF, Garssen B, Slot E, Berbee M, Berkhof J, Meezenbroek Ede J, Slieker W, Visser A, Meijer S, Beelen RH: Breast cancer surgery-induced immunomodulation. J Surg Oncol 2010, 102(6):640-8.

20. de Kruijf EM, van Nes JG, Sajet A, Tummers QR, Putter H, Osanto S, Speetjens FM, Smit VT, Liefers GJ, van de Velde CJ, Kuppen PJ: The predictive value of HLA class I tumor cell expression and presence of intratumoral Tregs for chemotherapy in patients with early breast cancer. Clin Cancer Res 2010, 16:1272-80.

21. Lavado-Valenzuela R, Benavides M, Carabantes F, Alonso A, Caballero A: MHC class I chain-related gene A transmembrane polymorphism in Spanish women with breast cancer. Tissue Antigens 2009, 74:46-9.

22. Ishigami S, Natsugoe S, Nakajo A, Arigami T, Kitazono M, Okumura H, Matsumoto M, Uchikado Y, Setoyama T, Sasaki K, Aikou T: HLA-class I expression in gastric cancer. J Surg Oncol 2008, 97:605-608.

23. Cordon-Cardo C, Fuks Z, Drobnjak M, Moreno C, Eisenbach L, Feldman M: Expression of HLA-A,B,C antigens on primary and metastatic tumor cell populations of human carcinomas. Cancer Res 1991, 51:6372-6380.

24. Madjd Z, Spendlove I, Pinder SE, Ellis IO, Durrant LG: Total loss of MHC class I is an independent indicator of good prognosis in breast cancer. Int J Cancer 2005, 117:248-55.

25. Sato N, Hirohashi Y, Tsukahara T, Kikuchi T, Sahara H, Kamiguchi K Ichimiya S, Tamura Y, Torigoe T: Molecular pathological approaches to human tumor immunology. Pathology International 2009, 59:205-217.

26. Mizukami $Y$, Kono K, Maruyama T, Watanabe M, Kawaguchi $Y$, Kamimura $K$ Fujii H: Downregulation of HLA Class I molecules in the tumour is associated with a poor prognosis in patients with oesophageal squamous cell carcinoma. Br J Cancer 2008, 99:1462-1467.

27. Zia A, Schildberg FW, Funke I: MHC class I negative phenotype of disseminated tumor cells in bone marrow is associated with poor survival in ROMO breast cancer patients. Int I Cancer 2001, 93:566-570.

28. Aptsiauri N, Cabrera T, Mendez R, Garcia-Lor A, Ruiz-Cabello F, Garrido F: Role of altered expression of HLA class I molecules in cancer progression. Adv Exp Med Biol 2007, 601:123-131.

\section{Pre-publication history}

The pre-publication history for this paper can be accessed here: http://www.biomedcentral.com/1471-2407/11/454/prepub

doi:10.1186/1471-2407-11-454

Cite this article as: Kaneko et al:: Clinical implication of HLA class I expression in breast cancer. BMC Cancer 2011 11:454 